\title{
Recreating the Face - A Mini-Review of Current Studies on 3D Bioprinting in Oral and Maxillofacial Surgery
}

\author{
Rwik Sen, $P h D^{*}$ \\ Active Motif, Inc., California, USA
}

\begin{abstract}
According to the United States National Institute of Dental and Craniofacial Research, craniofacial anomalies are the most common birth defects. Genetic, epigenetic, and environmental causes lead to craniofacial anomalies which can range from cleft lip and palate to major defects in the development of the skull, face, brain, eyes, ears, and nose. Oral and maxillofacial surgeries are performed on individuals with craniofacial defects, but success of surgery is dependent on nature of the defect. Some defects are too complex which need multiple surgeries, but still not be completely cured by traditional methods. In this direction, 3 dimensional or 3D bioprinting has emerged as a promising modern technology that can significantly benefit the field. A personalized medicine approach to address craniofacial defects is provided by 3D-printing technologies which integrate doctors, engineers and researchers to work for a common goal [1]. Hence, this mini review of literature on 3D bioprinting discusses the technology in the context of craniofacial, and hence oral and maxillofacial therapeutics. In the field of oral and maxillofacial therapeutics, researchers and surgeons are aiming to develop a 3D scaffold by direct 3D printing technology to fabricate complicated tissue grafts. They need to possess all the necessary biological properties and environment for cell division and tissue regeneration. Hence, the current and future challenges of 3D bioprinting will be to achieve the above criteria is a cost-effective and timely manner.
\end{abstract}

\section{Introduction}

3D bioprinting has addressed the problems associated with traditional surgeries and therapy. The emergence of 3D printing technology occurred in the 1990's when synthetic inks were used to generate fabricating scaffolds, leading up to the invention of bioprinting in the $21^{\text {st }}$ century [2]. Bioprinting employs bioinks which are composed of biocompatible substances made of cells or matrices derived from natural sources which have applications in tissue engineering $[3,4]$. The technology evolved from additive manufacturing where biomaterials are used to develop scaffolds which precisely fit into the dimensions of a craniofacial defect (Figure 1). There are several advantages of 3D bioprinting in oral and maxillofacial surgery since traditional methods like bone grafting are not always convenient. This is because several different kinds of bones and cartilages, derived from various progenitor stem cells, are complexly organized to form the craniofacial skeleton. In this direction, 3D bioprinting has immensely improved the field of craniofacial surgeries because it allows for the selection of specific kinds of cells to bio print the target tissue. Another very important advantage of $3 \mathrm{D}$ bioprinting is that success of the craniofacial surgery is no longer solely dependent on the surgeon. Further, 3D printing adopts a personalized therapeutic approach by recapitulating the physical and aesthetic properties of the patient's target tissue in the prostheses, which was labor-intensive earlier [1]. Hence, 3D bioprinting makes the process of oral and maxillofacial surgeries significantly convenient, greatly improves their outcome, and enhances the quality of life of patients [5].

\section{The Process of 3D Bioprinting}

The process of 3D bioprinting can be categorized into pre-bioprinting, bioprinting, and post-bioprinting [6-8]. At the very beginning, suitable cells for synthesizing the bioink are isolated and cultured to obtain a large amount of viable starting material.

In the pre-bioprinting step, imaging is performed on the tissue that will be bioprinted. The imaging is done by technology like Computed tomography (CT) or cone beam CT in Digital Imaging and Communications in Medicine (DICOM) format and magnetic resonance $[6,8,9]$. Standard Triangle Language (STL) format is used to provide the image as input to the bioprinter $[6,8,9]$. Hence, a Computer Aided Design (CAD) model of the target tissue is obtained [6].

*Corresponding author: Rwik Sen, PhD, Active Motif, Inc., 1914 Palomar Oaks Way, Suite 150, Carlsbad, California 92008, USA

Accepted: December 29, 2020

Published online: December 31, 2020

Citation: Sen R (2020) Recreating the Face - A Mini-Review of Current Studies on 3D Bioprinting in Oral and Maxillofacial Surgery. Archives Oral Maxillofac Surg 3(1):86-89 


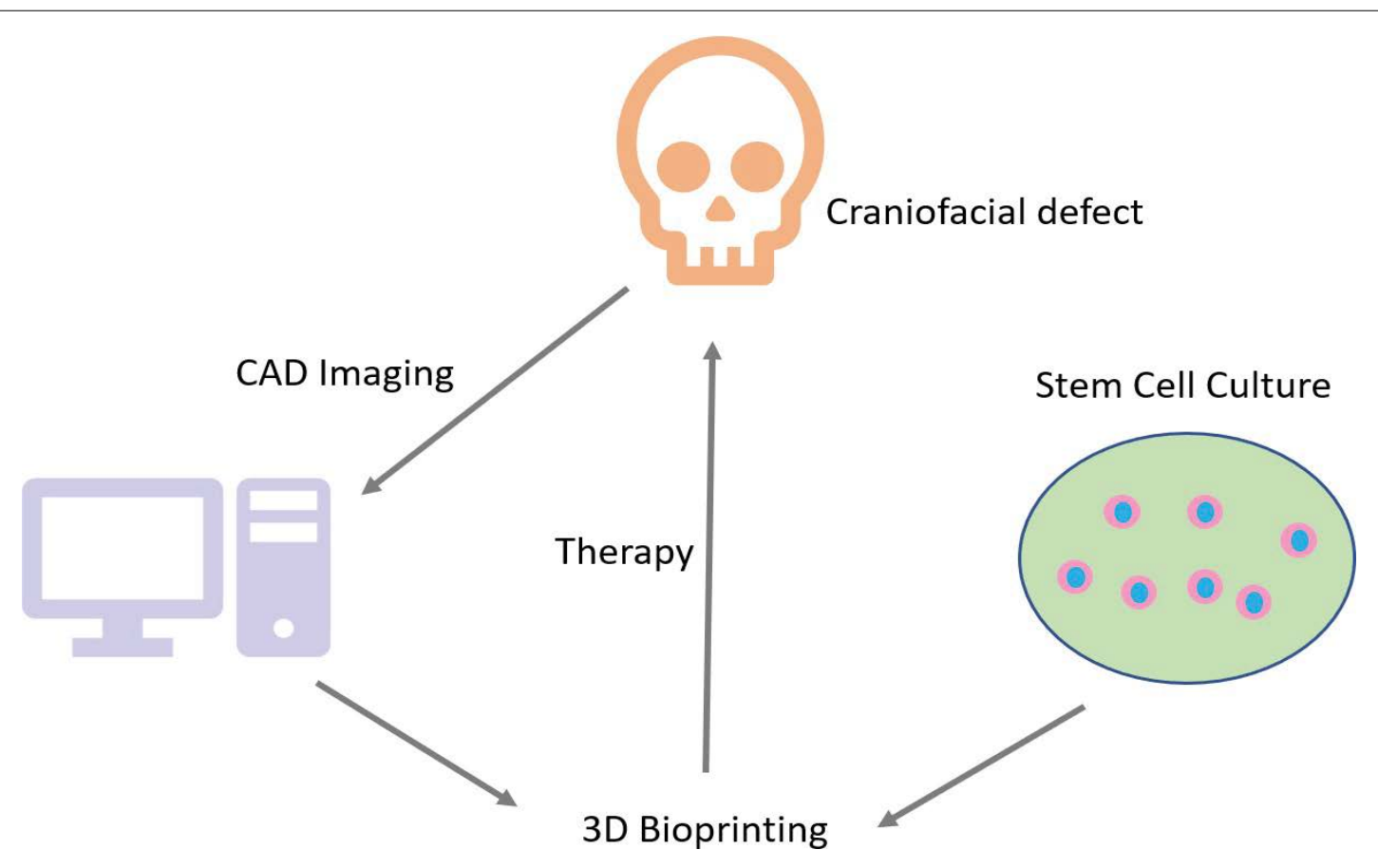

Figure 1: Schematic outline of 3D Bioprinting. The damaged tissue is imaged by CAD, followed by bioprinting of the image using bioink that are derived of cells and other factors. Next, the bioprinted tissue is surgically placed in the patient as a therapy for repair, reconstruction, regeneration, or protheses.

The bioprinting process performs a layer after layer printing of the tissue image that is acquired in the previous step, using the bioink made of cells, nutrients, and matrix loaded onto the printer cartridge [6]. The bioprinted cell-based entity is called pre-tissue which is moved into an incubator for maturation [10]. Following maturation, cells are apportioned onto the biocompatible scaffold in a layer after layer manner in succession leading to the development of 3D biological constructs that resemble tissues [6].

Post-bioprinting contributes to the process of developing and maintaining the mechanical stability and functionality of the 3D construct [8]. Cells in the bioprinted entity need mechanical and chemical signals for remodeling and development of tissues for proper sustainability of the construct. In this direction, bioreactors provide the ideal environment and simulations that are necessary for the tissue to survive, mature and vascularize $[6,7]$.

\section{D Bioprinting in Oral, Maxillofacial, and Facial Reconstructive Surgery}

In oral, maxillofacial, and facial reconstructive surgery, the technology of 3D bioprinting encompasses certain criteria like developing prototypes of facial anatomy, improving contour symmetry of the face after surgery, bioprinting pre-contoured grafts, developing superior quality prostheses for patients having scars, asymmetry, and malformations [11]. Another area to apply 3D bioprinting technology in surgery is the development of advanced simulation models for medical students [11]. Using imaging techniques like CT and magnetic resonance imaging (MRI), anatomical scans are prepared and saved in standard format like DICOM. Next, CAD software is used to generate virtual 3-dimensional prototypes with STL for 3D bioprinting where biomaterial is laid in patterned layers to achieve the ultimate conformation [11]. A recent review of 3D bioprinting in maxillofacial surgery reported 297 publications from 35 countries where 2889 patient outcomes were improved using 3D bioprinting [12]. These publications show that highest number of clinical indications occur for dental implantations and reconstruction of the mandible [12]. Overall, 3D bioprinting leads to improved surgery in terms of precision and reduced time, but high costs and production time remain as disadvantages [12].

Further applications of 3D bioprinting in craniofacial surgeries include trauma surgery, orthognathic surgery, facial prosthetics, Temporo Mandibular Joint (TMJ) and complex facial reconstruction [13-18]. 3D bioprinting shows several benefits in the above areas. In trauma surgery, 3D printed titanium mesh cures postoperative enophthalmos or diplopia caused by inefficient orbital wall reconstruction. 3D bioprinting solves issues caused by blowout fractures of orbital floor and walls [16]. 3D bioprinting helps in precision diagnosis during orthognathic surgeries. 3D bioprinting has several advantages over traditional prosthesis. A common problem in TMJ reconstruction is called autorotation which is an instability of condyle and fossa of TMJ that affects proper placement of maxilla. Personalized orthognathic surgical guide (POSG) system helps solved this problem using 3D bioprinting [19]. Advantages of 3D bioprinting in complex facial reconstruction include accurate plate adaptation, precise harvest of bones, low bone-plate distance and blood loss, and reduced time of surgery and anesthesia $[13,20]$. The implications of 3D bioprinting are extensively discussed by Dr. Devid Zille in his article. Dr. Zille leads the Patient-Specific Implant initiative for Osteomed, which is among the world's largest small-bone implant manufacturers for Maxillofacial, Neuro, and Extremities surgery. 


\section{Craniofacial applications of 3D bioprinting}

Successful biomaterials for craniofacial reconstruction need to be biocompatible, easy to print, enable conduction and induction of signals specific to bone and cartilage cells, and show mechanical properties that resemble craniofacial bones and cartilages [6]. Some of the materials that have shown promise as craniofacial scaffolds are bio-ceramics, hydrogels, polymers, and their composites [21]. Consequently, personalized therapy for craniofacial reconstruction by $3 D$ bioprinting has been achieved with high fidelity for precise defects [6]. In this direction, the success of 3D bioprinting is a great boon because bones and cartilages are among tissue that need to be transplanted with highest frequency due to their losses from trauma, osteoporosis, tumors, and other causes.

\section{Methodologies for 3D Bioprinting}

Inkjet printing, laser-assisted, and extrusion are some methods of 3D bioprinting. They have been reviewed in detail $[2,22]$. Inkjet printing has been employed in bone regeneration for regulating in vitro osteoblast differentiation and in vivo osteogenesis. The processes were observed using bone morphogenetic protein-2 (BMP-2) which was applied on a microporous scaffold of Derma Matrix, collagen, and fibronectin [23]. Inkjet was also successful in printing biodegradable osteoconductive calcium phosphate scaffolds and collagen coating [24]. Laser-assisted methods like selective laser sintering (SLS) have successfully repaired and regenerated craniofacial bones, periodontal and osteochondral defects using various polymers [2]. Fused deposition modeling (FDM) is an extrusion-based process which has shown success in rodent osteogenic differentiation of dental pulp stem cells and bone formation [25].

\section{Considerations for scaffolds}

In addition to favorable culture conditions in the bioink, the cells used in the bioink depend on certain characteristics of the scaffold to deliver the function desired from them. One such scaffold characteristic is surface topography. The differentiation of mesenchymal stem cells (MSCs) into osteoblast and form new tissue are maximum if the surfaces are concave, and it tends to minimum as the surface changes from smooth to convex [26]. Osteogenic potential of wavy scaffolds are higher as seen from calcium deposition, high alkaline phosphatase activity and osteocalcin staining [27]. Concave surfaces also favor cell adhesion by integrin which is responsible for non-nuclear signal transduction to promote osteogenic gene expression [28].

Another scaffold characteristic is pore size. Efficient bone engineering through osteogenesis and angiogenesis is favored by hypoxic conditions that are achieved through pore sizes in the range of $100-300 \mu \mathrm{m}$ or higher [29]. A gradient of higher oxygen at the peripheries and hypoxia at the center achieved through a variation in pore sizes along the scaffold is seen to favor MSC differentiation and hence, bone tissue engineering [30,31].

\section{Conclusion}

Although promising and successful so far, the process of $3 \mathrm{D}$ bioprinting still require considerable development to be applied on a large scale to patients. Further research is required to expand its applications to a wider range of therapeutics. Nonetheless, 3D bioprinting has shown success in reconstructive, repair, and protheses related to oral and maxillofacial surgery. Advances in stem cell research, superior engineering, and computational techniques will further advance this elegant technology. At the regulatory level, care needs to be taken to ensure proper administration, affordability, accessibility, and health insurance amenability for this technology.

\section{References}

1. Nyberg EL, Farris AL, Hung BP, et al. (2017) 3D- printing technologies for craniofacial rehabilitation, reconstruction and regeneration. Ann Biomed Eng 45: 45-57.

2. Tao O, Kort-Mascort J, Lin Y, et al. (2019) The applications of 3D Printing for Craniofacial Tissue Engineering. Micromachines (Basel) 10: 480 .

3. Brian Derby (2012) Printing and prototyping of tissues and scaffolds. Science 338: 921-926.

4. Nichol JW, Khademhosseini A (2009) Modular tissue engineering: Engineering biological tissues from the bottom-up. Soft Matter 5: 1312-1319.

5. Salah M,Tayebi L, Moharamzadeh K, et al. (2020) Three-dimensional bio-printing and bone tissue engineering: technical innovations and potential applications in maxillofacial reconstructive surgery. Maxillofac Plast Reconstr Surg 42: 18.

6. Dwivedi R, Mehrotra D (2020) 3D bioprinting and craniofacial regeneration. J Oral Biol Craniofac Res 10: 650-659.

7. Ozbolat IT (2015) Bioprinting scale-up tissue and organ constructs for transplantation. Trends Biotechnol 33: 395-400.

8. Shafiee A, Atala A (2016) Printing technologies for medical applications. Trends Mol Med 22: 254-265.

9. Mironov V, Boland T, Trusk T, et al. (2003) Organ printing: Computer-aided jet-based 3D tissue engineering. Trends Biotechnol 21: 157-161.

10. Thomas DJ (2016) Could 3D bioprinted tissues offer future hope for microtia treatment? Int J Surg 32: 43-44.

11. Aldaadaa A, Owji N, Knowles J (2018) Three-dimensional Printing in Maxillofacial Surgery: Hype versus Reality. J Tissue Eng 9: 2041731418770909.

12. Louvrier A, Marty P, Barrabe A, et al. (2017) How useful is 3D printing in maxillofacial surgery? J Stomatol Oral Maxillofac Surg 118: 206-212.

13. Keyhan SO, Ghanean S, Navabazam A, et al. (2016) Three-dimensional printing: A novel technology for use in oral and maxillofacial operations. A Textbook of Advanced Oral and Maxillofacial Surgery 3.

14. Noureldin MG, Dessoky NY (2020) 3D Printing: Towards the future of oral and maxillofacial surgery acta scientific dental sciences 4: 107-112.

15. Ruiz-Cantu L, Gleadall A, Faris C, et al. (2020) Multi-material 3D bioprinting of porous constructs for cartilage regeneration. Mater Sci Eng C Mater Biol Appl 109: 2019.110578. 
16. Sigaux N, Pourchet L, Breton P, et al. (2019) 3D Bioprinting: principles fantasies and prospects. J Stomatol Oral Maxillofac Surg 120: 128-132.

17. VanKoevering K K, Zopf DA, Hollister SJ (2019) Tissue engineering and 3-dimensional modeling for facial reconstruction. Facial Plast Surg Clin North Am 27: 151-161.

18. Visscher DO, Bos E J, van Zuijlen PP (2015) 3D bioprinting of cartilage: Challenges concerning the reconstruction of a burned ear. Ned Tijdschr Geneeskd 160: A9698.

19. Oberoi G, Nitsch S, Edelmayer M, et al. (2018) 3D printing-encompassing the facets of dentistry. Front Bioeng Biotechnol 6: 172.

20. Ujam A, Gardner O, Gomez EC, et al. (2019) 3D Bioprinting cartilage for facial reconstruction. British Journal of Oral and Maxillofacial Surgery 57: E88-E89.

21. Zhang L, Yang G, Johnson BN, et al. (2019) Three-dimensional (3D) printed scaffold and material selection for bone repair. Acta Biomater 84: 16-33.

22. Kacarevic ZP, Rider PM, Alkildani S, et al. (2018) An Introduction to 3D bioprinting: possibilities, challenges and future aspects. Materials (Basel) 11: 2199.

23. Cooper GM, Miller ED, Decesare GE, et al. (2010) Inkjet-based biopatterning of bone morphogenetic protein-2 to spatially control calvarial bone formation. Tissue Eng Part A 16: 1749-1759.

24. Inzana JA, Olvera D, Fuller SM, et al. (2014) 3D printing of composite calcium phosphate and collagen scaffolds for bone regeneration. Biomaterials 35: 4026-4034.
25. Li J, Chen M, Wei X, et al. (2017) Evaluation of 3D-printed polycaprolactone scaffolds coated with freeze-dried platelet-rich plasma for bone regeneration. Materials (Basel) 10: 831.

26. Graziano A, d'Aquino R, Cusella-De Angelis MG, et al. (2008) Scaffold's surface geometry significantly affects human stem cell bone tissue engineering J Cell Physiol 214: 166-172.

27. Ji S, Guvendiren M (2019) 3D printed wavy scaffolds enhance mesenchymal stem cell osteogenesis micromachines (Basel) 11: 31.

28. Olivares-Navarrete R, Rodil SE, Hyzy SL, et al. (2015) Role of integrin subunits in mesenchymal stem cell differentiation and osteoblast maturation on graphitic carbon-coated microstructured surfaces. Biomaterials 51: 69-79.

29. Karageorgiou V, Kaplan D (2005) Porosity of 3D biomaterial scaffolds and osteogenesis. Biomaterials 26: 5474-5491.

30. Abbasi N, Ivanovski S, Gulati K, et al. (2020) Role of offset and gradient architectures of 3-D melt electrowritten scaffold on differentiation and mineralization of osteoblasts. Biomaterials Research 24.

31. Di Luca A, Ostrowska B, Lorenzo-Moldero I, et al. (2016) Gradients in pore size enhance the osteogenic differentiation of human mesenchymal stromal cells in three-dimensional scaffolds. Sci Rep 6: 22898. 